\title{
Clinical characteristics and outcome of HIV infected patients with chronic kidney disease in Sub Saharan Africa: an example from Cameroon
}

Marie Patrice Halle ${ }^{1,2^{*}}$, Noel Essomba ${ }^{1}$, Hilaire Djantio ${ }^{3}$, Germaine Tsele ${ }^{1}$, Hermine Fouda ${ }^{2,4}$, Namme Henri Luma ${ }^{2,4}$, Enow Gloria Ashuntantang ${ }^{4,5}$ and Folefack Francois Kaze ${ }^{4}$

\begin{abstract}
Background: Chronic kidney disease (CKD) is one of the major complications of Human immune deficiency Virus (HIV) and a risk factor for poor outcome of these patients. We aimed to describe the profile and outcome of HIV positive patients with CKD in Douala general hospital in Cameroon.
\end{abstract}

Methods: HIV positive patients with CKD referred to the nephrologist from January 2007 to March 2013 were included. Socio demographic, clinical (history and stage of HIV, comorbidities, baseline nephropathy, used of c-ART), para clinical data at referral (serum urea, creatinine, full blood count, CD4 count, serum calcium, phosphorus, albumin), dialysis initiation and outcome at 1 year were collected from medical records. GFR was estimated using Chronic Kidney Disease Epidemiology Collaboration (CKD-EPI) equations. CKD was defined and classified according to the Kidney Disease Improving Global Outcomes (KDIGO 2012).

Results: We included 156 patients (51.3\% men) with a mean age of $45.4 \pm 12.1$ years. Hypertension (36.5\%), diabetes (17.9\%) and Hepatitis C (7.7\%) were the main comorbidities. HIV associated nephropathy (27.6\%), chronic glomerulonephritis (15.4\%) diabetes (14.1\%) and hypertension (13.5\%) were the leading causes of kidney disease. Before referral HIV status was known by 109 (69.9\%) patients, with 76 (69.7\%) being on c-ART. Median $\mathrm{CD}_{4}$ count was 241 (117-438) cells $/ \mathrm{mm}^{3}$. Prevalence of anemia (93.9\%), hypocalcemia (68.6\%) and Proteinuria (77.6\%) was high, 94 (60.3\%) patients were at CKD stage 5 at referral and 37 (23.7\%) underwent emergency dialysis. After 1 year, 64 (41.0\%) patients were lost to follow up. The mortality rate was 49\% and 25 (28.7\%) were maintenance hemodialysis, and being on c-ART was associated with a lower risk of death (HR: $0.45 ; 95 \% \mathrm{Cl}: 0.23-0.89 ; p=0.021$ ).

Conclusion: HIV patients with CKD were referred late with high morbidity and need for urgent hemodialysis. HIVAN was the main etiology of CKD and mortality rate was high mainly due to the absence of C-ART at referral.

Keywords: Chronic kidney disease, Human immune deficiency virus, Douala, Cameroon

\footnotetext{
* Correspondence: patricehalle@yahoo.fr

${ }^{1}$ Faculty of Medicine and Pharmaceutical Sciences, University of Douala,

Douala, Cameroon

²Department of Internal Medicine, Douala General Hospital, PO Box: 4856,

Douala, Cameroon

Full list of author information is available at the end of the article
}

(c) The Author(s). 2019 Open Access This article is distributed under the terms of the Creative Commons Attribution 4.0 International License (http://creativecommons.org/licenses/by/4.0/), which permits unrestricted use, distribution, and reproduction in any medium, provided you give appropriate credit to the original author(s) and the source, provide a link to the Creative Commons license, and indicate if changes were made. The Creative Commons Public Domain Dedication waiver (http://creativecommons.org/publicdomain/zero/1.0/) applies to the data made available in this article, unless otherwise stated. 


\section{Background}

The number of people living with human immunodeficiency virus (HIV) is increasing, with 36.9 million people infected in 2015 and 2 million news cases per year [1]. The introduction of combined antiretroviral therapy (cART) has improved the lifespan of HIV infected patients and exposed them to various organs damage [2]. Chronic kidney disease (CKD) is one of the major complications amongst HIV infected patients, with a prevalence ranging from 3.5 to $48.5 \%$ [2-5] and the presence of CKD is a risk factor for mortality of these patients [6-8].

CKD in HIV can be related to the virus itself or no. Common disease due to the virus are HIV associated nephropathy (HIVAN) and HIV-immune complex nephropathy (HIVICK). Black people from African origin are more prone to HIVAN due to predisposing genetic polymorphismus $[9,10]$ and the risk is 3 to 6 fold higher compared to white $[11,12]$. HIVAN was the 3th leading cause of end stage kidney disease (ESKD) amongst black in the United States of America [13, 14]. The introduction of c-ART has reduced the incidence of HIVAN and ESKD [3, 15], but CKD remain a serious problem in these patients. Others HIV-related factors are low CD4 counts, high viral load, hepatitis $\mathrm{C}$ virus co-infection and some antiretroviral drugs such as Tenofovir, Indinavir, Lopinavir/Ritonavir, Atazanavir/Ritonavir [11, 16-19]. Other reported causes of CKD not HIV related are older age, diabetes mellitus and hypertension $[4,5,7,20,21]$. In a study of Jung et al. in Germany, HIVICK (26.1\%), nephroangiosclerosis and diabetic nephropathy (20.3\% respectively) were the leading causes of CKD in HIV positive patients [22] while Wyatt et al. and Onen et al. reported hypertensive renal disease as the most common kidney disease [23, 24]. In Spain, diabetic nephropathy was the etiology of ESKD in $14 \%$ of HIV-positive patients [25].

Early identification of kidney disease and early referral to the nephrologist gives a chance to implement treatments that slow the progression of kidney dysfunction and reduces the need of renal replacement therapy (RRT) $[26,27]$ and therefore reduces mortality of patients.

The prevalence and incidence of HIV in Sub-Saharan Africa (SSA) is the highest in the world $(75.8 \%$ of new cases) with greatest mortality rate $[28,1]$. Kidney disease is frequent amongst these patients in SSA with a pooled prevalence of $14.6 \%$ [29]. In Cameroon a country in SSA, HIV is the 5th leading cause of ESKD with a prevalence of 10.8 to $13.5 \%$ amongst patients on maintenance hemodialysis [30-32]. Late referral of CKD patients to the nephrologist is common in that setting and outcome of patients is poor in general and survival of HIV infected patients on RRT is lower compared to their negative counterpart [8, 33, 34]. Studies on HIV patients with non-dialyzed CKD are rare in SSA. The aim of this study was to describe the profile and outcome of HIV positive patients with CKD in Cameroon, a country where patients have free access to c-ART.

\section{Methods \\ Study setting}

We conducted a retrospective study in the nephrology outpatient consultation of the Douala general hospital, the main referral tertiary hospitals for patients with kidney disease in the littoral region with a capacity of 320 beds. At the time of the study, the center operated with one internist- nephrologist and two general practitioners. Each patient has a medical file that is opened at the first consultation. In Cameroon, c-ART is highly subsidized by the government since 2007and access to treatment is not limited. HIV patients with CKD are followed up by internist-nephrologist with a special training in management of HIV related diseases. The study was authorized by the Director of the Douala general hospital and ethical approval was obtained from the Douala University Ethics Committee.

\section{Patients}

All HIV positive patients aged 18 years and above with CKD referred to the nephrologist from 1 January 2007 to 31th March 2013 were included. HIV patients with missing relevant data were excluded. Data were collected from medical records and included socio demographic (age, sex, marital status), clinical (history and stage of HIV, comorbidities, baseline nephropathy, used of cART before referral), para clinical data at referral (serum urea, creatinine, full blood count, CD4 count, serum calcium, phosphorus, albumin), dialysis initiation and outcome at 1 year (death, loss of follow up, alive, on dialysis).

\section{Definition of operational terms}

Estimated glomerular filtration rate (e GFR, $\mathrm{mL} / \mathrm{min}$ ) was computed using Chronic Kidney Disease Epidemiology Collaboration (CKD-EPI) equations [35].

CKD was defined and classified according to the Kidney Disease Improving Global Outcomes (KDIGO 2012) [36]. eGFR was therefore classified as follow: G1 (eGFR 290); G2 (eGFR 60-89); G3a (eGFR 45-59); G3b (eGFR 30-44); G4 (eGFR 15-29) and G5 (eGFR < 15).

ESKD was considered in any patient with eGFR $<15 \mathrm{ml} /$ $\mathrm{min} / 1.73 \mathrm{~m}^{2}$ and on maintenance hemodialysis for more than 3 months. Hypertension was defined as a blood pressure $>140 / 90 \mathrm{mmh}$ or evidence from records that the patient was on antihypertensive treatment. Diabetes was considered if a reported history of diagnostic of this condition or use of glucose lowering 1 agents, or fasting blood sugar above $1.26 \mathrm{~g} / \mathrm{ml}$. The background nephropathy was made by the nephrologist and mainly based on clinical arguments in the absence of renal histology data. 
HIVAN was considered if a CKD patient presented with nephrotic range proteinuria with or without edema, normal blood pressure, hyperechogenic kidney with normal size on ultrasound.

Lost to follow up was considered in any patient who was recorded at the start of the study period, and become lost within the first year of follow up, with no data on the outcome.

\section{Statistical analysis}

Data were analyzed using the software Statistical Package for Social Sciences (SPSS Inc., Chicago, USA) version 23.0. Qualitative variables were described as frequency and percentages and compared with Chi-square test or Fisher's exact test. Quantitative variables with normal distribution were described as mean \pm standard deviation (SD); skewed data as median and inter quartile range (IQR). Their comparison was done respectively with Student T-test and Mann Whitney U test. One year mortality rate was calculated by dividing the number of deceased patients by the number of patients whose outcome was known at 1 year (alive or death). Predictors of death were assessed using Cox proportional hazard regression models. We adjusted the basic models for age and sex. Survival curves of patients treated or not with c-ART at referral were compared using Kaplan Meier estimator (Log rank test). Statistical significance was set at a $p$ value $<0.05$.

\section{Results}

\section{Baseline characteristics}

A total of 156 patients were included in this study with $80 / 156(51.3 \%)$ men. Mean age of participants was $45.4 \pm 12.1$ years and women were younger $(p=0.002)$. Mean age wasn't significantly different across stage of kidney disease $(p=0.39)$. Their baseline characteristics are shown in Table 1. Hypertension (36.5\%), diabetes (17.9\%) and hepatitis $\mathrm{C}(7.7 \%)$ were the main comorbidities. HIVAN 43/156 (27.6\%), chronic glomerulonephritis 24/156 (15.4\%) diabetes 22/156 (14.1\%), and hypertension $21 / 156(13.5 \%)$ were the leading causes of kidney disease. In $24.4 \%$ of cases the etiology was unknown. Before referral 109/156 (69.9\%) patients knew their HIV status of which only 76/109 (69.7\%) were already on cART. Median $\mathrm{CD}_{4}$ count was $241(117-438)$ cells $/ \mathrm{mm}^{3}$, with no difference between genders $(p=0.619)$ or CKD stage $(p=0.456)$

\section{Biological profile}

Biologic data of study participants are reported in Table 2. Most patients (58.3\%) had CKD stage 5 at referral. Blood Urea Nitrogen and Creatinine significantly increase with stage of CKD $(p<0.001)$. Median GFR was $10.1 \mathrm{ml} / \mathrm{min} / 1.73 \mathrm{~m}^{2}$ overall and was lower in women compare to men $(p=0.019)$ and decreases with stage of
CDK $(p<0.001)$. Proteinuria was present in $121(77.6 \%)$ patients, hematuria in $42(26.9 \%)$, leucocyturia in 23 (14.7\%) and glycosuria in $10(6.4 \%)$ patients with no statistically difference between genders or stage of kidney disease. Mean hemoglobin level was $8.2 \pm 2.3 \mathrm{~g} / \mathrm{dl}$ overall and lower in women $(p=0.019)$ and decreases with stage of CKD $(p=0.004)$. Anemia was frequent with a prevalence of $93.9 \%(90.7 \%$ in men, $97.3 \%$ in women, $p=$ $0.167)$ and was more common in late stage of CKD; from $75 \%$ in stage $3 a$ to $97.7 \%$ in stage $5(p=0.017)$. Mean calcemia was $84.6 \pm 13.7 \mathrm{mg} / \mathrm{l}$ and lower in women $(p=0.024)$ and did not varies significantly across stage of CKD $(p=0.413)$. Hypocalcemia concerned 70/102 (68.6\%) with no difference neither across gender nor stage of disease. Phosphoremia increases with stage of CKD with a borderline $p$ value (0.056). Hyperphosphoremia was seen in 32/89 (36.0\%) patients and its frequency increases with stage of CKD (16.7\% in stage 3a vs $45.2 \%$ in stage 5). The distribution of other hematologic parameters, electrolytes, lipid profile showed no major differences across gender or stage of CKD.

\section{Outcome}

In total, 37/156 (23.7\%) patients initiated dialysis immediately at referral and $34 / 37$ (91.9\%) on a temporary central venous catheter. Indication for dialysis were: uremic syndrome (81.1\%), acute pulmonary edema (16.2\%), hyperkalemia (10.8\%), and uremic encephalopathy $(8.1 \%)$ (Table 3). After 1 year of follow up, 64 patients (41\%) were lost to follow up and $5(3.2 \%)$ were transferred to another nephrology unit (Table 3). For those with known outcome at 1 year $(87 / 156)$ the overall mortality rate was $49.4 \%$ (43/87 patients) and was similar between both sex $(p=0.914)$, and $19(21.8 \%)$ patients were on chronic hemodialysis. As shown in Table 4, outcome after 1 year of follow up varied according to the stage of kidney disease with respectively $40,33.3,45.5$ and $15.4 \%$ of patient of stage $3 \mathrm{a}, 3 \mathrm{~b}, 4$ and stage 5 being alive and not on chronic dialysis with a borderline $p$ value $(0.085)$. The rate of patients lost to follow up varied across CKD stages: stage $3 \mathrm{a}(61.5 \%), 3 \mathrm{~b}(70.0 \%)$ and $4(62.5 \%)$ than in stage $5(24.2 \%)$ of disease $(<0.001)$.

Age and sex adjusted Cox regression analysis showed that being on c-ART at referral was associated with a lower risk of death in the first year of follow up; Hazard Ratio: 0.45 ; 95\% CI: 0.23-0.89; $p=0.021$ (Table 5). Kaplan Meier estimator confirm this observation, with a better survival curve in patients already on c-ART at referral. ( $p=0.021$ for Log Rank test) Fig. 1 .

\section{Discussion}

This study on the characteristic of HIV positive patients with CKD referred to a tertiary hospital in SSA showed that included patients were young adults, with others 
Table 1 General characteristics of the study population at referral

\begin{tabular}{|c|c|c|c|c|c|c|c|c|c|}
\hline \multirow[t]{2}{*}{ Variables } & \multirow[t]{2}{*}{ Total } & \multicolumn{2}{|l|}{ Gender } & \multirow[t]{2}{*}{$P$} & \multicolumn{4}{|l|}{ Stage } & \multirow[t]{2}{*}{$P$} \\
\hline & & Male & Female & & G3a & G3b & G4 & G5 & \\
\hline $\mathrm{N}(\%)$ & $156(100.0)$ & $80(51.3)$ & $76(48.7)$ & & $13(8.3)$ & $20(12.8)$ & $32(20.5)$ & $91(58.3)$ & \\
\hline Age (years), Mean $\pm S D^{a}$ & $45.4 \pm 12.1$ & $48.3 \pm 11.8$ & $42.4 \pm 11.6$ & 0.002 & $46.6 \pm 14.9$ & $49.0 \pm 12.8$ & $43.2 \pm 12.7$ & $45.3 \pm 11.2$ & 0.394 \\
\hline Age (years), Min-Max & $22-82$ & $25-82$ & $22-72$ & & $26-72$ & $28-70$ & $25-82$ & $22-77$ & \\
\hline \multicolumn{10}{|l|}{ Marital status, n (\%) } \\
\hline Married & $87(55.8)$ & $58(72.5)$ & $29(38.2)$ & & $9(69.2)$ & $9(45.0)$ & $17(53.1)$ & $52(57.1)$ & \\
\hline Single & $53(34.0)$ & $17(21.3)$ & $36(47.4)$ & & $3(23.1)$ & $8(40.0)$ & $13(40.6)$ & $29(31.9)$ & \\
\hline Divorced & $2(1.3)$ & $1(1.3)$ & $1(1.3)$ & & $0(0.0)$ & $0(0.0)$ & $1(3.1)$ & $1(1.1)$ & \\
\hline Widow & $14(9.0)$ & $4(5.0)$ & $10(13.2)$ & $<0.001$ & $1(7.7)$ & $3(15.0)$ & $1(3.1)$ & $9(9.9)$ & 0.784 \\
\hline \multicolumn{10}{|l|}{ Employment status, n (\%) } \\
\hline Unemployed & $56(35.9)$ & $11(13.8)$ & $45(59.2)$ & & $6(46.2)$ & $4(20.0)$ & $12(37.5)$ & $34(37.4)$ & \\
\hline Public agent & $10(6.4)$ & $8(10.0)$ & $2(2.6)$ & & $0(0.0)$ & $1(5.0)$ & $1(3.1)$ & $8(8.8)$ & \\
\hline Private sector & $37(23.7)$ & $29(36.3)$ & $8(10.5)$ & & $5(38.5)$ & $5(25.0)$ & $8(25.0)$ & $19(20.9)$ & \\
\hline Informal sector & $39(25.0)$ & $18(22.5)$ & $21(27.6)$ & & $1(77)$ & $6(300)$ & $8(25.0)$ & $24(26.4)$ & \\
\hline Retired & $14(9.0)$ & $14(17.5)$ & $0(0.0)$ & $<0.001$ & $1(7.7)$ & $6(30.0)$ & $8(25.0)$ & $24(26.4)$ & 0.548 \\
\hline \multicolumn{10}{|l|}{ Co morbidity, n (\%) } \\
\hline Hypertension & $57(36.5)$ & 25 (31.6) & $32(42.1)$ & 0.177 & $4(33.3)$ & $6(30.0)$ & $10(31.3)$ & $37(40.7)$ & 0.690 \\
\hline Diabetes & $28(17.9)$ & $18(22.8)$ & $10(13.2)$ & 0.119 & $2(16.7)$ & $6(30.0)$ & $7(21.9)$ & $13(14.3)$ & 0.372 \\
\hline $\mathrm{HCV}^{\mathrm{b}}$ coinfection & $12(7.7)$ & $7(8.8)$ & $5(6.6)$ & 0.611 & $1(7.7)$ & $2(10.0)$ & $1(3.1)$ & $8(8.8)$ & 0.742 \\
\hline Chronic use of NSAID ${ }^{c}$ & $9(5.8)$ & $4(5.1)$ & $5(6.6)$ & 0.744 & $1(8.3)$ & $1(5.0)$ & $1(3.1)$ & $6(6.6)$ & 0.876 \\
\hline HBV ${ }^{d}$ coinfection & $6(3.8)$ & $5(6.3)$ & $1(1.3)$ & 0.211 & $0(0.0)$ & $2(10.0)$ & $3(9.4)$ & $1(1.1)$ & 0.070 \\
\hline \multicolumn{10}{|c|}{ Background nephropathy, n (\%) } \\
\hline HIVAN & $43(27.6)$ & $26(32.5)$ & $17(22.4)$ & & $4(30.8)$ & $5(25.0)$ & $6(18.8)$ & $28(30.8)$ & \\
\hline $\begin{array}{l}\text { Chronic } \\
\text { glomerulonephritis }\end{array}$ & $24(15.4)$ & $9(11.3)$ & $15(19.7)$ & & $1(7.7)$ & $5(25.0)$ & $5(15.6)$ & $13(14.3)$ & \\
\hline Diabetes & $22(14.1)$ & $15(18.8)$ & $7(9.2)$ & & $1(7.7)$ & $6(30.0)$ & $4(12.5)$ & $11(12.1)$ & \\
\hline Hypertension & $21(13.5)$ & $7(8.8)$ & $14(18.4)$ & & $3(23.1)$ & $2(10.0)$ & $12(37.5)$ & $21(23.1)$ & \\
\hline $\begin{array}{l}\text { Chronic interstitial } \\
\text { nephritis }\end{array}$ & $7(4.5)$ & $3(3.8)$ & $4(5.3)$ & & $1(7.7)$ & $0(0.0)$ & $1(3.1)$ & $5(5.5)$ & \\
\hline Polykystosis & $1(0.6)$ & $1(1.3)$ & $0(0.0)$ & & $0(0.0)$ & $1(5.0)$ & $0(0.0)$ & $0(0.0)$ & \\
\hline Unknown & $38(24.4)$ & $19(23.8)$ & $19(25.0)$ & 0.139 & $3(23.1)$ & $2(10.0)$ & $12(37.5)$ & $21(23.1)$ & 0.242 \\
\hline Known HIV infected, n (\%) & $109(69.9)$ & $56(70.0)$ & $53(69.7)$ & 0.971 & $8(61.5)$ & $16(80.0)$ & $21(65.6)$ & $64(70.3)$ & \\
\hline $\begin{array}{l}\text { HIV infection vintage } \\
\text { (month) })^{g}\end{array}$ & $36(11-96)$ & $36(12-108)$ & $\begin{array}{l}36(10.3- \\
96)\end{array}$ & 0.856 & $24(1-144)$ & $15(4-48)$ & $24(7.5-60)$ & $36(12-108)$ & 0.251 \\
\hline Use of CART $^{\mathrm{f}}, \mathrm{n}(\%)$ & $76(48.7)$ & $36(45.0)$ & $40(52.6)$ & 0.340 & $7(53.8)$ & $10(50.0)$ & $11(34.4)$ & $48(52.7)$ & \\
\hline Duration on $C A R T^{f g}$ & $36(6.8-96)$ & 36 (12-99) & $36(6-96)$ & 0.879 & $\begin{array}{l}40(18.3- \\
90)\end{array}$ & $8(2-79.5)$ & $48(4-72)$ & $36(9.8-105)$ & 0.797 \\
\hline \multicolumn{10}{|l|}{ Drug regimen, $\mathrm{n}(\%), n=76$} \\
\hline 1st line & $65(85.5)$ & $33(91.7)$ & $32(80.0)$ & & $6(85.7)$ & $8(80.0)$ & $8(72.7)$ & $43(89.6)$ & \\
\hline $2^{\text {nd }}$ line & $11(14.5)$ & $3(8.3)$ & $8(20.0)$ & 0.199 & $1(14.3)$ & $2(20.0)$ & $3(27.3)$ & $5(10.4)$ & \\
\hline $\mathrm{CD}_{4}$ count $^{9}, n=88$ & $\begin{array}{l}241(117- \\
438)\end{array}$ & $\begin{array}{l}256(138- \\
387)\end{array}$ & $\begin{array}{l}216(96- \\
535)\end{array}$ & 0.619 & $\begin{array}{l}101(66- \\
376)\end{array}$ & $\begin{array}{l}176.5(137- \\
333.3)\end{array}$ & $\begin{array}{l}278(117.3- \\
549)\end{array}$ & $\begin{array}{l}271(120- \\
451)\end{array}$ & 0.456 \\
\hline
\end{tabular}

Values in italics are significant $(p$ value $<0.05)$

${ }^{\mathrm{a}} S D$ Standard deviation, ${ }^{\mathrm{b}} H C V$ Hepatitis $\mathrm{C}$ virus, ${ }^{\mathrm{C}}$ NSAID Non-steroidal anti-inflammatory drugs, ${ }^{\mathrm{d}} H B V$ Hepatitis B virus, ${ }^{\mathrm{e}} H I V A N$ Human immuno-deficiency virus associated nephropathy, ${ }^{\mathrm{f}} \mathrm{CART}$ Combined antiretroviral treatment; ${ }^{\mathrm{g}}$ Median (1st-3th quartiles) 
Table 2 Biologic profile and stage of chronic kidney disease of study participants at referral

\begin{tabular}{|c|c|c|c|c|c|c|c|c|c|}
\hline \multirow[t]{2}{*}{ Biologic Data } & \multirow[t]{2}{*}{ Total } & \multicolumn{2}{|l|}{ Gender } & \multirow[t]{2}{*}{ P } & \multicolumn{4}{|l|}{ Stage } & \multirow[t]{2}{*}{$P$} \\
\hline & & Male & Female & & G3a & G3b & G4 & G5 & \\
\hline Blood urea nitrogen $(\mathrm{g} / \mathrm{l})^{\mathrm{h}}$ & $1.12(0.7-2.1)$ & $1.1(0.7-2.0)$ & $1.3(0.7-2.2)$ & 0.918 & $0.4(0.3-0.6)$ & $0.7(0.5-1.2)$ & $0.8(0.6-1.1)$ & $1.8(1.05-2.5)$ & $\begin{array}{l}< \\
0.001\end{array}$ \\
\hline Serum Creatinine $(\mathrm{mg} / \mathrm{l})^{\mathrm{h}}$ & $\begin{array}{l}67.7(29.3- \\
118.5)\end{array}$ & $\begin{array}{l}73.4(29.6- \\
101.3)\end{array}$ & $\begin{array}{l}61.9(28.4- \\
135.1)\end{array}$ & 0.457 & $\begin{array}{l}16.7(15.8- \\
19.6)\end{array}$ & $\begin{array}{l}24.7(21- \\
25.4)\end{array}$ & $\begin{array}{l}33.9(29.6- \\
40.6)\end{array}$ & $\begin{array}{l}103(79.8- \\
160.9)\end{array}$ & $\begin{array}{l}< \\
0.001\end{array}$ \\
\hline $\mathrm{GFR}^{\mathrm{a}}\left(\mathrm{ml} / \mathrm{min} / 1.73 \mathrm{~m}^{2}\right)^{\mathrm{h}}$ & $10.1(4.7-27.2)$ & $10.2(6.5-31.5)$ & $9.5(3.4-23.3)$ & 0.028 & $\begin{array}{l}49.5(47.9- \\
51.8)\end{array}$ & $\begin{array}{l}37.1(31.9- \\
40.5)\end{array}$ & $\begin{array}{l}22.4(19.8- \\
26.51)\end{array}$ & $5.3(3.2-8.3)$ & $\begin{array}{l}< \\
0.001\end{array}$ \\
\hline CKD ${ }^{\mathrm{b}}$ stage, $\mathrm{n}(\%)$ & & & & & - & - & - & - & \\
\hline G1 & $0(0.0)$ & $0(0.0)$ & $0(0.0)$ & & - & - & - & - & \\
\hline G2 & $0(0.0)$ & $0(0.0)$ & $0(0.0)$ & & - & - & - & - & \\
\hline G3a & $13(8.3)$ & $8(10.0)$ & $5(6.6)$ & & - & - & - & - & \\
\hline G3b & $20(12.8)$ & $13(16.3)$ & $7(9.2)$ & & - & - & - & - & \\
\hline G4 & $32(20.5)$ & $17(21.3)$ & $15(19.7)$ & & - & - & - & - & \\
\hline G5 & $91(58.3)$ & $42(52.5)$ & $49(64.5)$ & 0.383 & - & - & - & - & \\
\hline \multicolumn{10}{|l|}{ Urinary dipstick, n (\%) } \\
\hline Proteinuria & $121(77.6)$ & $65(81.3)$ & $56(73.7)$ & 0.258 & $7(53.8)$ & $15(75.0)$ & $27(84.4)$ & $72(79.1)$ & 0.154 \\
\hline Hematuria & $42(26.9)$ & $26(32.5)$ & $16(21.1)$ & 0.107 & $2(15.4)$ & $5(25.0)$ & $5(15.6)$ & $30(33.3)$ & 0.183 \\
\hline Leucocyturia & $23(14.7)$ & $10(12.5)$ & $13(17.1)$ & 0.417 & $1(7.7)$ & $3(15.0)$ & $3(9.4)$ & $16(17.8)$ & 0.594 \\
\hline Glycosuria & $10(6.4)$ & $5(6.3)$ & $5(6.6)$ & 0.933 & $0(0.0)$ & $1(5.0)$ & $2(6.3)$ & $7(7.8)$ & 0.746 \\
\hline \multicolumn{10}{|l|}{ Lipid profile, Mean \pm SD } \\
\hline Total cholesterol (g/l) & $1.8 \pm 0.6$ & $1.7 \pm 0.5$ & $2.0 \pm 0.7$ & 0.109 & $1.7 \pm 0.3$ & $1.9 \pm 0.5$ & $2.1 \pm 0.7$ & $1.8 \pm 0.6$ & 0.551 \\
\hline $\mathrm{LDL}^{\mathrm{C}}$ cholesterol (g/l) & $1.0 \pm 0.6$ & $1.0 \pm 0.6$ & $1.1 \pm 0.7$ & 0.668 & $0.4 \pm 0.2$ & $0.3 \pm 0.2$ & $0.5 \pm 0.2$ & $0.4 \pm 0.2$ & 0.214 \\
\hline $\mathrm{HDL}^{\mathrm{d}}$ cholesterol $(\mathrm{g} / \mathrm{l})$ & $0.4 \pm 0.2$ & $0.3 \pm 0.1$ & $0.4 \pm 0.2$ & 0.064 & $0.6 \pm 0.2$ & $1.0 \pm 0.1$ & $1.1 \pm 0.7$ & $1.0 \pm 0.7$ & 0.825 \\
\hline Triglycerides (g/l) & $1.8 \pm 1.1$ & $1.6 \pm 0.8$ & $2.0 \pm 1.3$ & 0.317 & $2.3 \pm 0.2$ & $1.6 \pm 2.1$ & $1.7 \pm 0.9$ & $1.9 \pm 0.9$ & 0.857 \\
\hline $\begin{array}{l}\text { Hemoglobin }(\mathrm{g} / \mathrm{dl}) \\
\text { Mean } \pm \text { SD }\end{array}$ & $8.2 \pm 2.3$ & $8.7 \pm 2.4$ & $7.6 \pm 1.9$ & 0.002 & $9.8 \pm 3.3$ & $9.0 \pm 2.2$ & $8.5 \pm 2.2$ & $7.7 \pm 2.0$ & 0.004 \\
\hline Anemia, $n=148, n(\%)$ & $139(93.9)$ & $68(90.7)$ & $71(97.3)$ & 0.167 & $9(75.0)$ & $18(90.0)$ & $29(93.5)$ & $84(97.7)$ & 0.017 \\
\hline \multicolumn{10}{|l|}{$\operatorname{MCV}^{e}(f \mathrm{l}), n=107$} \\
\hline Mean \pm SD & $85.5 \pm 9.8$ & $85.9 \pm 9.4$ & $85.1 \pm 10.2$ & 0.674 & $85.5 \pm 9.8$ & $85.8 \pm 8.1$ & $85.3 \pm 7.9$ & $85.4 \pm 10.7$ & 0.999 \\
\hline$<80$ & $34(31.8)$ & $17(31.5)$ & $17(32.1)$ & & $3(50.0)$ & $4(30.8)$ & $6(30.0)$ & $21(30.9)$ & \\
\hline$>100$ & $8(7.5)$ & $4(7.4)$ & $4(7.5)$ & 0.997 & $0(0.0)$ & $0(0.0)$ & $1(5.0)$ & $7(10.3)$ & 0.765 \\
\hline \multicolumn{10}{|l|}{$\mathrm{MCH}^{\mathrm{f}}(\mathrm{pg}), n=100$} \\
\hline Mean \pm SD & $28.1 \pm 4.3$ & $28.0 \pm 5.0$ & $28.1 \pm 3.5$ & 0.885 & $29.2 \pm 3.6$ & $28.6 \pm 3.8$ & $27.6 \pm 3.3$ & $28.3 \pm 4.0$ & 0.791 \\
\hline$<28$ & $48(48.0)$ & $22(45.8)$ & $26(50.0)$ & & $3(50.0)$ & $6(46.2)$ & $11(57.9)$ & $28(45.2)$ & \\
\hline$>32$ & $15(15.0)$ & $8(16.7)$ & $7(13.5)$ & 0.875 & $2(33.3)$ & $2(15.4)$ & $2(10.5)$ & $9(14.5)$ & 0.793 \\
\hline \multicolumn{10}{|l|}{$\left.\operatorname{WBC}^{\mathrm{g}}(\mathrm{G} /)^{\mathrm{h}}\right)^{\mathrm{h}}, n=124$} \\
\hline $\begin{array}{l}\text { Median (1st-3th } \\
\text { quartiles) }\end{array}$ & $5.3(4.1-7.1)$ & $5.3(4.4-7.2)$ & $5.1(3.9-7.1)$ & 0.276 & $4.9(4.2-5.3)$ & $5.3(3.8-8.8)$ & $5.2(4-8)$ & $5.4(4.1-7.1)$ & 0.843 \\
\hline$<4$ & $25(20.7)$ & $9(15.0)$ & $16(26.2)$ & & $0(0.0)$ & $5(31.3)$ & $5(21.7)$ & $15(20.3)$ & \\
\hline$>10$ & $12(9.9)$ & $5(8.3)$ & $7(11.5)$ & 0.218 & $0(0.0)$ & $1(6.3)$ & $1(4.3)$ & $10(13.5)$ & 0.314 \\
\hline \multicolumn{10}{|l|}{ Platelets $(G / l), n=121$} \\
\hline Mean \pm SD & $223.4 \pm 94.1$ & $214.6 \pm 93.0$ & $232.4 \pm 95.2$ & 0.300 & $224.3 \pm 112.5$ & $213.5 \pm 85.4$ & $214.4 \pm 91.6$ & $223.6 \pm 93.6$ & 0.877 \\
\hline$<150$ & $25(20.7)$ & $16(26.2)$ & $9(15.0)$ & 0.267 & $2(22.2)$ & $2(11.1)$ & $5(20.8)$ & $16(22.5)$ & 0.511 \\
\hline \multicolumn{10}{|l|}{ Natremia $(\mathrm{mmol} / \mathrm{l}), n=111$} \\
\hline Mean \pm SD & $135.0 \pm 8.8$ & $134.6 \pm 9.1$ & $135.4 \pm 8.6$ & 0.639 & $136.1 \pm 6.5$ & $134.9 \pm 6.1$ & $136.8 \pm 10.1$ & $134.3 \pm 9.2$ & 0.718 \\
\hline$<135$ & $44(39.6)$ & $23(40.4)$ & $21(38.9)$ & 0.571 & $5(55.6)$ & $5(38.5)$ & $4(19.0)$ & $31(44.9)$ & 0.393 \\
\hline
\end{tabular}


Table 2 Biologic profile and stage of chronic kidney disease of study participants at referral (Continued)

\begin{tabular}{|c|c|c|c|c|c|c|c|c|c|}
\hline \multirow[t]{2}{*}{ Biologic Data } & \multirow[t]{2}{*}{ Total } & \multicolumn{2}{|l|}{ Gender } & \multirow[t]{2}{*}{$P$} & \multicolumn{4}{|l|}{ Stage } & \multirow[t]{2}{*}{$P$} \\
\hline & & Male & Female & & G3a & G3b & G4 & G5 & \\
\hline \multicolumn{10}{|c|}{ Potassium (mmol/l), $n=109$} \\
\hline Mean \pm SD & $5.3 \pm 3.3$ & $5.0 \pm 0.98$ & $4.9 \pm 1.2$ & 0.726 & $4.2 \pm 0.5$ & $4.8 \pm 0.9$ & $4.8 \pm 0.8$ & $5.1 \pm 1.2$ & 0.096 \\
\hline$<3.5$ & $4(3.7)$ & $2(3.6)$ & $2(3.7)$ & & $0(0.0)$ & $0(0.0)$ & $1(5.0)$ & $3(4.3)$ & \\
\hline$>5.0$ & $47(43.1)$ & $27(49.1)$ & $20(37.0)$ & 0.437 & $1(11.1)$ & $6(50.0)$ & $10(50.0)$ & $30(43.5)$ & 0.435 \\
\hline \multicolumn{10}{|c|}{ Chloremia (mmol/l), $n=101$} \\
\hline Mean \pm SD & $101.7 \pm 9.4$ & $101.0 \pm 9.7$ & $102.6 \pm 8.9$ & 0.410 & $99.1 \pm 10.3$ & $103.8 \pm 11.5$ & $103.1 \pm 7.2$ & $101.3 \pm 9.4$ & 0.631 \\
\hline$<95$ & $25(24.8)$ & $14(25.5)$ & $11(23.9)$ & & $3(37.5)$ & $3(25.0)$ & $3(17.6)$ & $16(24.6)$ & \\
\hline$>105$ & $33(32.7)$ & $15(27.3)$ & $18(39.1)$ & 0.421 & $2(25.0)$ & $6(50.0)$ & $5(29.4)$ & $20(30.6)$ & 0.724 \\
\hline \multicolumn{10}{|c|}{ Calcemia $(\mathrm{mg} / \mathrm{l}), n=102$} \\
\hline Mean \pm SD & $84.6 \pm 13.7$ & $87.5 \pm 15.3$ & $81.5 \pm 11.1$ & 0.024 & $84.6 \pm 8.8$ & $89.9 \pm 10.5$ & $87.5 \pm 10.0$ & $83.1 \pm 15.1$ & 0.413 \\
\hline$<90$ & $70(68.6)$ & $31(60.8)$ & $39(76.5)$ & & $5(62.5)$ & $5(55.6)$ & $8(47.1)$ & $52(75.4)$ & \\
\hline$>105$ & $2(2.0)$ & $2(3.9)$ & $0(0.0)$ & 0.128 & $0(0.0)$ & $1(11.1)$ & $0(0.0)$ & $1(1.4)$ & 0.102 \\
\hline \multicolumn{10}{|c|}{ Phosphoremia (mg/l), $n=89$} \\
\hline Mean \pm SD & $56.6 \pm 31.5$ & $50.8 \pm 26.4$ & $62.3 \pm 35.1$ & 0.086 & $38.2 \pm 19.3$ & $36.6 \pm 8.5$ & $49.9 \pm 28.2$ & $62.1 \pm 33.2$ & 0.056 \\
\hline$<35$ & $22(24.7)$ & $12(27.3)$ & $10(22.2)$ & & $2(33.3)$ & $5(71.4)$ & $5(35.7)$ & $10(16.1)$ & \\
\hline$>55$ & $32(36.0)$ & $11(25.0)$ & $21(46.7)$ & 0.096 & $1(16.7)$ & $0(0.0)$ & $3(21.4)$ & $28(45.2)$ & 0.022 \\
\hline \multicolumn{10}{|c|}{ Uricemia (mg/l), $n=66$} \\
\hline Mean \pm SD & $89.3 \pm 32.2$ & $91.7 \pm 33.2$ & $88.7 \pm 31.4$ & 0.530 & $78.1 \pm 16.1$ & $78.5 \pm 24.2$ & $88.4 \pm 35.2$ & $93.8 \pm 34.2$ & 0.509 \\
\hline$>70$ & $50(75.8)$ & $27(79.4)$ & $23(71.9)$ & 0.515 & $5(83.3)$ & $6(75.0)$ & $11(73.3)$ & $28(75.7)$ & 0.982 \\
\hline \multicolumn{10}{|c|}{ Albuminemia $(\mathrm{g} / \mathrm{l}), n=80$} \\
\hline Mean \pm SD & $32.8 \pm 11.2$ & $33.5 \pm 13.9$ & $31.9 \pm 7.5$ & 0.531 & $34.0 \pm 2.9$ & $29.7 \pm 7.2$ & $37.3 \pm 18.4$ & $31.7 \pm 9.0$ & 0.294 \\
\hline$<35$ & $51(63.7)$ & $27(65.9)$ & $24(61.5)$ & 0.688 & $3(60.0)$ & $6(75.0)$ & $9(56.3)$ & $34(65.4)$ & 0.822 \\
\hline
\end{tabular}

Values in italics are significant $(p$ value $<0.05)$

${ }^{a} G F R$ Glomerular filtration rate (estimated using MDRD formulae), ${ }^{b} C K D$ Chronic kidney disease, ${ }^{c} L D L$ Low density lipoprotein, ${ }^{d} H D L$ High density lipoprotein, $M C V$ Mean corpuscular volume, $M C H^{f}$ Mean corpuscular haemoglobin, $W B C^{g}$ White blood cells; ${ }^{\text {h}}$ Median (1st-3th quartiles)

risks factors of CKD such as hypertension, diabetes and HVC. Proteinuria was the main urinary abnormality affecting more than $3 / 4$ of patients. At referral median CD4 was low and less than half of the study population were on c-ART. HIVAN was the main presumed etiology of CKD. Patients were referred at advanced stage of CKD for the majority with various biological abnormalities among witch anemia was the most frequent. Dialysis was initiated in almost $1 / 4$ patients at referral due to life threatening conditions. At 1 year, the rate of loss of follow up and mortality was very high and absence of c$\mathrm{ART}$ at referral was the main predictor of death.

CKD is epidemic among HIV-infected populations especially in Africa [29]. The prevalence of HIV in SSA is the highest in the world and kidney disease is frequent amongst these patients with a pooled prevalence of $14.6 \%[28,29]$. In the present study patients were young adult with mean age of 45 years and males represented half of the study population. It's well known that CKD and HIV affects more young adults in SSA [37-39]. Contrary to our finding CKD affect more male due to genetic and environmental factor. One explanation to our finding is that HIV is more prevalent in women in our setting and therefore increase the number of women with CKD [37, 39, 40].

HIV infected patients often have one or more risk factors for CKD [7, 41, 42]. In the present study others non-HIV related risk factors were hypertension, diabetes, Hepatitis $\mathrm{B}$ and $\mathrm{C}$ infection. HIVAN was the leading cause of CKD (27.6\%). This is in accordance with most studies in populations from African origin $[38,39,43,44]$. Our reported rate is similar to the results of Okpechi et al. in South Africa and Da Silva et al. in Brazil $[45,46]$. In the contrary Jung et al. in Germany found that HIVICK (26.1\%) was the main etiology of CKD in HIV patients. This is due to racial difference as it is well known that HIVICK affect more white people [47]. The introduction of c-ART has reduced the incidence of HIVAN and ESKD in the world [15]. In Cameroon, patients have free access to c-ART. The prevalence of HIVAN found in this study remain high and possible 
Table 3 Outcome of the study population after 1 year of follow up

\begin{tabular}{|c|c|c|c|c|}
\hline Outcome & $\begin{array}{l}\text { Total } \\
n(\%) \\
N=156\end{array}$ & $\begin{array}{l}\text { Male } \\
n(\%) \\
N=80\end{array}$ & $\begin{array}{l}\text { Female } \\
\mathrm{n}(\%) \\
N=76\end{array}$ & $P$ \\
\hline \multicolumn{5}{|l|}{ At referral } \\
\hline Dialysis & $37(23.7)$ & $16(20.0)$ & $21(27.6)$ & 0.263 \\
\hline \multicolumn{5}{|l|}{ Vascular access } \\
\hline$C V C^{a}$ & $34(91.9)$ & $16(100.0)$ & $18(85.7)$ & \\
\hline$A V F^{b}$ & $3(8.1)$ & $0(0.0)$ & $3(14.3)$ & 0.243 \\
\hline \multicolumn{5}{|l|}{ Dialysis indication, $n=37$} \\
\hline Uremic syndrome & $30(81.1)$ & $12(75.0)$ & $18(85.7)$ & 0.437 \\
\hline Acute pulmonary edema & $6(16.2)$ & $3(18.8)$ & $3(14.3)$ & $>0.999$ \\
\hline Severe hyperkalemia & $4(10.8)$ & 212.5) & $2(9.5)$ & $>0.999$ \\
\hline Uremic encephalopathy & $3(8.1)$ & $1(6.3)$ & $2(9.5)$ & $>0.999$ \\
\hline \multicolumn{5}{|l|}{ At 1 year } \\
\hline \multicolumn{5}{|l|}{ Unknown } \\
\hline Transfer to another hospital & $5(3.2)$ & $3(3.8)$ & $2(2.6)$ & 0.692 \\
\hline Lost to follow up & $64(41.0)$ & $34(42.5)$ & $30(39.5)$ & 0.701 \\
\hline \multicolumn{5}{|l|}{ Known, $n=87$} \\
\hline Deceased & $43(49.4)$ & $21(48.8)$ & $22(50.0)$ & 0.914 \\
\hline On chronic dialysis & $25(28.7)$ & $12(27.9)$ & $13(29.5)$ & 0.866 \\
\hline Alive and not on dialysis & $19(21.8)$ & $10(23.3)$ & $9(20.5)$ & 0.752 \\
\hline $\mathrm{CD}^{c}, n=21$ & $380(265-598.5)$ & 353.5 (251.5-588.8) & $476(271.5-685)$ & 0.804 \\
\hline
\end{tabular}

${ }^{\mathrm{a}}$ CVC Central venous catheter, ${ }^{\mathrm{b}}$ AVF Arteriovenous fistulae; ${ }^{\mathrm{c}}$ Median (1st-3th quartiles)

raisons could be: the ignorance of the HIV status $(30.1 \%$ at referral), the severity of the immune depression at referral (low median CD4 count), and the mostly the absence of c-ART for the majority of patients (51.3\%) at referral. Others presumed etiology of CKD in this study were diabetes, chronic glomerulonephritis and hypertension. This is in accordance with reported studies [7, 20-25].

Our patients were referred at advanced stage of CKD with $60 \%$ at stage 5 with various clinical and biological complications. Consequently urgent dialysis without preparation was initiated in some patients at referral. Late referral is a serious problem in developing countries in general and in Cameroon in particular with high morbidity, emergency dialysis and poor outcome [33, 34, 48]. Early identification of kidney disease and early referral to the nephrologist gives a chance to implement treatments that slow the progression of kidney dysfunction and reduce the need of RRT and mortality of patients [26, 27].

Table 4 Outcome by stage of kidney disease after 1 year of follow up

\begin{tabular}{|c|c|c|c|c|c|}
\hline \multirow[t]{2}{*}{ Outcome } & \multicolumn{4}{|c|}{ Stage of kidney disease } & \multirow[t]{2}{*}{$P$} \\
\hline & $\begin{array}{l}\text { G3a } \\
N=13\end{array}$ & $\begin{array}{l}\text { G3b } \\
N=20\end{array}$ & $\begin{array}{l}G 4 \\
N=32\end{array}$ & $\begin{array}{l}\mathrm{G} 5 \\
N=91\end{array}$ & \\
\hline \multicolumn{6}{|l|}{ Unknown } \\
\hline Transfer to another hospital & $0(0)$ & $0(0)$ & $1(3.1)$ & $4(4.4)$ & 0.680 \\
\hline Lost to follow up & $8(61.5)$ & $14(70.0)$ & $20(62.5)$ & $22(24.2)$ & $<0.001$ \\
\hline Known & $n=5$ & $n=6$ & $n=11$ & $n=65$ & \\
\hline Deceased & $2(40.0)$ & $3(50.0)$ & $4(36.4)$ & $34(52.3)$ & 0.766 \\
\hline On chronic dialysis & $1(20.0)$ & $1(16.7)$ & $2(18.2)$ & $21(32.3)$ & 0.656 \\
\hline Alive and not on dialysis & $2(40.0)$ & $2(33.3)$ & $5(45.5)$ & $10(15.4)$ & 0.085 \\
\hline
\end{tabular}

Values in italics are significant $(p$ value $<0.05)$ 
Table 5 Predictive factors of mortality (Cox regression analysis)

\begin{tabular}{|c|c|c|c|c|}
\hline \multirow[t]{2}{*}{ Variable } & \multicolumn{2}{|l|}{ Basic models } & \multicolumn{2}{|l|}{ Final models } \\
\hline & $\mathrm{HR}^{\mathrm{a}}\left(95 \% \mathrm{Cl}^{\mathrm{b}}\right)$ & $p$ & $\mathrm{HR}^{\mathrm{a}}\left(95 \% \mathrm{Cl}^{\mathrm{b}}\right)$ & $p$ \\
\hline Age (per years increase) & $0.99(0.96-1.02)$ & 0.452 & $1.00(0.97-1.02)$ & 0.742 \\
\hline Gender (female vs male) & $1.02(0.55-1.89)$ & 0.963 & $1.15(0.61-2.16)$ & 0.663 \\
\hline Unemployed & $1.37(0.68-2.73)$ & 0.380 & & \\
\hline Hypertension & $1.58(0.84-2.96)$ & 0.156 & & \\
\hline Diabetes & $0.56(0.23-1.40)$ & 0.216 & & \\
\hline Hepatitis B & $0.61(0.08-4.53)$ & 0.632 & & \\
\hline Hepatitis C & $1.43(0.58-3.55)$ & 0.442 & & \\
\hline On $\mathrm{CART}^{\mathrm{C}}$ & $0.45(0.23-0.89)$ & 0.021 & $0.45(0.23-0.89)$ & 0.021 \\
\hline CD4 count (cells $/ \mathrm{mm}^{3}$ ) & $1.00(0.99-1.00)$ & 0.823 & & \\
\hline \multicolumn{5}{|l|}{ Stage of CKD at arrival } \\
\hline G3 & 1 (reference) & & & \\
\hline G4 & $0.45(0.10-2.03)$ & 0.299 & & \\
\hline G5 & $1.16(0.42-3.17)$ & 0.780 & & \\
\hline Hemoglobin level (per g/dl) & $0.91(0.79-1.05)$ & 0.201 & & \\
\hline Calcium level (per 10 mg/l) & $0.81(0.59-1.10)$ & 0.178 & & \\
\hline Creatinine level (per 10 mg/l) & $1.01(0.97-1.05)$ & 0.606 & & \\
\hline
\end{tabular}

Basic models are adjusted for age and sex; final models are adjusted for age, sex and all predictors with a $p$ value $<0.1$ in the basic models (use of cART) Values in italics are significant $(p$ value $<0.05)$

${ }^{\mathrm{a}} \mathrm{HR}$ Hazard ratio, ${ }^{\mathrm{b}} \mathrm{Cl}$ Confidence interval, ${ }^{\mathrm{c}} \mathrm{CART}$ Combined anti-retroviral treatment

We found at 1 year that $41 \%$ of patients were lost to follow up. This is extremely high and a dangerous situation. It is recommended that patients should have a regular nephrology care when e GFR $<30 \mathrm{ml} / \mathrm{min} / 1.73$ [36]. Raisons for this situation in our setting could be: the silent evolution of CKD as many patients visit the hospital only when they feel symptoms. Also the lack of funds is a serious problem; $35 \%$ of our patients were unemployed in a country where medical insurance is almost inexistent and the cost of care high and out-of-



Fig. 1 Survival probability during follow-up from Kaplan Meier estimator comparing patients treated with (blue curve) and without (green curve) c-ART at referral; $p$ value $=0.021$ for Log Rank test 
pocket spending represent an important proportion [49]. Also the fear of dialysis is common.

HIV and CKD are two risk factor that expose affected patients to death $[50,51]$ For those with known outcome at 1 year in this study $(87 / 156)$ the overall mortality rate was high $(49 \%)$. This rate is higher compare to mortality of ESKD patients in the same setting $[8,34]$ Studies have reported renal dysfunction as predictor of death amongst HIV infected patients and the risk increases with the stage of CKD $[51,52]$. The main factor associated to death of our patients was the absence of c-ART at referral. This is in accordance with reported finding in the literature $[15,53,54]$. Similarly we reported recently in the same setting a lower survival rate of HIV patients on hemodialysis compared to negative one and the absence of c-ART was the main factor associated to death [8]. The use of c-ART and suppression of HIV RNA improved kidney function [55] and therefore reduce the risk of death. Studies have reported that earlier stage of the infection, higher CD4 counts, and treatment with c-ART was associated with better survival of HIV positive patients. [50, 56] Our patients were referred late. Current guidelines recommend specialist referral for patients with eGFR $<30 \mathrm{ml} / \mathrm{min} /$ $1.73 \mathrm{~m}^{2}$ [36]. Absent or late pre dialysis nephrology care is associated with increased morbidity and mortality $[33,57,58]$. There is a need in our setting to evaluate the determinants of referral pattern and develop strategies to improve the situation.

\section{Limitations}

We acknowledge some limitations to this study: first the retrospective design of the study with analysis based on data found in records. The absence of renal biopsy for the histological confirmation of the background nephropathy especially of HIVAN. But histological confirmation of HIVAN is infrequent in most studies from SSA and the diagnosis is usually made clinically. We did not have viral load and CD4 count for most patients during follow up, due to financial constraint as those test could are expensive and payment is out of pocket. Therefore the evaluation of control of the HIV infection was not possible. Also causes of death were not available. Data were collected from a single center, which could raise issues regarding the generalizability to the entire country. However, the study center is the only public institution to provide sufficient data for the range of time covered in the current study. This study has as major strength that it describe for the first time the baseline characteristics and outcome of HIV infected patients with CKD in Cameroon and in SSA. This could help to develop strategies to improve the care of these group of patients.

\section{Conclusion}

This study revealed that HIV infected patients with CKD are mostly young adult, they are referred at late stage of the disease, with high morbidity and need for urgent hemodialysis. HIVAN is the main etiology of CKD and mortality rate is high. Absence of c-ART at referral might be associated with an increase in patient's mortality. There is need to develop strategies for more multidisciplinary care of HIV infected patients and consequently improve their outcome.

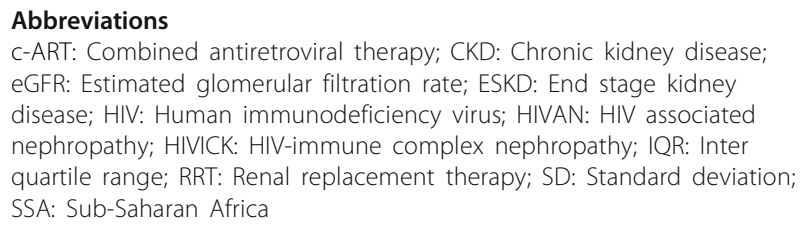

\section{Acknowledgements}

None.

\section{Authors' contributions}

MPH: Study design and conception, manuscript drafting. NE: Study design, supervision of data collection, critical revision of the manuscript. HD: Data analysis and interpretation, critical revision of the manuscript. GT: Data collection, and interpretation, critical revision of manuscript. HF: Supervision of data collection and critical revision of the manuscript. NHL: Supervision of data analysis and critical revision of the manuscript. EGA: Study conception and critical revision of the manuscript. FFK: Study design, critical revision of the manuscript. All authors have read and approve of the final manuscript.

\section{Funding}

None.

Availability of data and materials

The datasets generated and/or analyzed during the current study are available from the corresponding author on reasonable request.

Ethics approval and consent to participate

Ethical approval was obtained from the Douala University Ethics Committee. Consent to participate was required and not applicable. We retrospectively used medical records.

Consent for publication

Not applicable.

\section{Competing interests}

The authors declare that they have no competing interests.

\section{Author details}

${ }^{1}$ Faculty of Medicine and Pharmaceutical Sciences, University of Douala, Douala, Cameroon. ${ }^{2}$ Department of Internal Medicine, Douala General Hospital, PO Box: 4856, Douala, Cameroon. ${ }^{3}$ Higher Institute of Health Sciences, Université des Montagnes, Bangangte, Cameroon. ${ }^{4}$ Faculty of Medicine and Biomedical Sciences, University of Yaoundé I, Yaoundé, Cameroon. ${ }^{5}$ Department of Internal Medicine, Yaounde General Hospital, Yaoundé, Cameroon.

Received: 25 September 2018 Accepted: 1 July 2019

Published online: 09 July 2019

\section{References}

1. GBD 2015 HIV Collaborators. Estimates of global, regional, and national incidence, prevalence, and mortality of HIV, 1980-2015: the global burden of disease study 2015. Lancet HIV. 2016;3(8):e361-87. 
2. Mocroft A, Ledergerber B, Katlama C, Kirk O, Reiss P, d'Arminio Monforte A, et al. Decline in the AIDS and death rates in the EuroSIDA study: an observational study. Lancet. 2003;362(9377):22-9.

3. Lucas GM, Mehta SH, Atta MG, Kirk GD, Galai N, Vlahov D, et al. End-stage renal disease and chronic kidney disease in a cohort of African-American HIV-infected and at-risk HIV-seronegative participants followed between 1988 and 2004. AIDS. 2007;21(18):2435-43.

4. Cheung CY, Wong KM, Lee MP, Liu YL, Kwok H, Chung R, et al. Prevalence of chronic kidney disease in Chinese HIV-infected patients. Nephrol Dial Transplant. 2007;22(11):3186-90.

5. Yanagisawa N, Ando M, Ajisawa A, Imamura A, Suganuma A, Tsuchiya K, et al. Clinical characteristics of kidney disease in Japanese HIV-infected patients. Nephron Clin Pract. 2011;118(3):c285-91.

6. Sackoff JE, Hanna DB, Pfeiffer MR, Torian LV. Causes of death among persons with AIDS in the era of highly active antiretroviral therapy: New York City. Ann Intern Med. 2006;145(6):397-406.

7. Ryom L, Kirk O, Lundgren JD, Reiss P, Pedersen C, De Wit S, et al. Advanced chronic kidney disease, end-stage renal disease and renal death among HIV-positive individuals in Europe. HIV Med. 2013;14(8):503-8.

8. Halle MP, Edjomo AM, Fouda H, Djantio H, Essomba N, Ashuntantang GE. Survival of HIV infected patients on maintenance hemodialysis in Cameroon: a comparative study. BMC Nephrol. 2018;19:166.

9. Rostand SG, Kirk KA, Rutsky EA, Pate BA. Racial differences in the incidence of treatment for end-stage renal disease. N Engl J Med. 1982;306(21):1276-9.

10. Klag MJ, Whelton PK, Randall BL, Neaton JD, Brancati FL, Stamler J. Endstage renal disease in African-American and white men. 16-year MRFIT findings. JAMA. 1997;277(16):1293-8.

11. Jotwani V, Li Y, Grunfeld C, Choi Al, Shlipak MG. Risk factors for ESRD in HIVinfected individuals: traditional and HIV-related factors. Am J Kidney Dis. 2012;59(5):628-35.

12. Freedman BI, Soucie JM, Stone SM, Pegram S. Familial clustering of endstage renal disease in blacks with HIV-associated nephropathy. Am J Kidney Dis. 1999;34(2):254-8.

13. Ross MJ, Klotman PE. HIV-associated nephropathy. AIDS. 2004;18(8):1089-99.

14. Winston JA, Burns GC, Klotman PE. The human immunodeficiency virus (HIV) epidemic and HIV-associated nephropathy. Semin Nephrol. 1998;18(4):373-7.

15. Lucas GM, Eustace JA, Sozio S, Mentari EK, Appiah KA, Moore RD. Highly active antiretroviral therapy and the incidence of HIV-1-associated nephropathy: a 12-year cohort study. AIDS. 2004;18(3):541-6.

16. Stanifer JW, Jing B, Tolan S, Helmke N, Mukerjee R, Naicker S, et al. The epidemiology of chronic kidney disease in sub-Saharan Africa: a systematic review and meta-analysis. Lancet Glob Health. 2014;2(3):e174-81.

17. Ryom L, Mocroft A, Kirk O, Worm SW, Kamara DA, Reiss P, et al. Association between antiretroviral exposure and renal impairment among HIV-positive persons with normal baseline renal function: the D:A:D study. J Infect Dis. 2013;207(9):1359-69.

18. Scherzer R, Estrella M, Li Y, Choi Al, Deeks SG, Grunfeld C, et al. Association of tenofovir exposure with kidney disease risk in HIV infection. AIDS. 2012; 26(7):867-75.

19. Vallet-Pichard A, Pol S. Natural history and predictors of severity of chronic hepatitis $\mathrm{C}$ virus (HCV) and human immunodeficiency virus (HIV) COinfection. J Hepatol. 2006:44(1 Suppl):S28-34.

20. Achhra AC, Mocroft A, Ross MJ, Ryom L, Lucas GM, Furrer $H$, et al. Kidney disease in antiretroviral-naïve HIV-positive adults with high CD4 counts: prevalence and predictors of kidney disease at enrolment in the INSIGHT Strategic Timing of AntiRetroviral Treatment (START) trial. HIV Med. 2015; 16(Suppl 1):55-63.

21. Mocroft A, Kirk O, Gatell J, Reiss P, Gargalianos P, Zilmer K, et al. Chronic renal failure among HIV-1-infected patients. AIDS. 2007:21(9):1119-27.

22. Jung $\mathrm{O}$, Haack HS, Brodt H-R, Grützmacher P, Geiger H, Amann K, et al. Changing spectrum of renal disease in HIV infection. Dtsch Med Wochenschr 1946. 2013;138(38):1887-91.

23. Wyatt CM, Morgello S, Katz-Malamed R, Wei C, Klotman ME, Klotman PE, et al. The spectrum of kidney disease in patients with AIDS in the era of antiretroviral therapy. Kidney Int. 2009;75(4):428-34

24. Onen NF, Overton ET, Seyfried W, Stumm ER, Snell M, Mondy K, et al. Aging and HIV infection: a comparison between older HIV-infected persons and the general population. HIV Clin Trials. 2010;11(2):100-9.

25. Saracho R, Martín EE, Comas JF, Arcos E, Mazuecos AB, Gentil MG, et al. Clinical evolution of chronic renal patients with HIV infection in replacement therapy. Nefrologia. 2015;35(5):457-64.
26. Yamagata K, Iseki K, Nitta K, Imai H, lino Y, Matsuo S, et al. Chronic kidney disease perspectives in Japan and the importance of urinalysis screening. Clin Exp Nephrol. 2008;12(1):1-8.

27. Ripley E. Complementary effects of angiotensin-converting enzyme inhibitors and angiotensin receptor blockers in slowing the progression of chronic kidney disease. Am Heart J. 2009;157(6, Supplement):S7-16.

28. Dwyer-Lindgren L, Cork M, Sligar A, Steuben K, Wilson K, Provost N, et al. Mapping HIV prevalence in sub-Saharian Africa between 2000 and 2017. Nature. 2019;570(7760):189-93.

29. Ekrikpo UE, Kengne AP, Bello AK, Effa EE, Noubiap JJ, Salako BL, et al. Chronic kidney disease in the global adult HIV-infected population: a systematic review and meta-analysis. PLoS One. 2018;13(4):e0195443.

30. Halle MP, Takongue C, Kengne AP, Kaze FF, Ngu KB. Epidemiological profile of patients with end stage renal disease in a referral hospital in Cameroon. BMC Nephrol. 2015;16(1):59.

31. Halle M, Luma H, Temfack E, Vanessa T, Kaze F, Ashuntantang G, et al. Prevalence of hepatitis B surface antigen and anti-HIV antibodies among patients on maintenance haemodialysis in Douala, Cameroon. Health Sci Dis. 2013;1:14

32. Luma HN, Halle MP, Eloumou SAFB, Azingala F, Kamdem F, Donfack-Sontsa $\mathrm{O}$, et al. Seroprevalence of human immunodeficiency virus, hepatitis $\mathrm{B}$ and $C$ viruses among haemodialysis patients in two newly opened centres in Cameroon. Pan Afr Med J. 2017;27:235.

33. Halle MP, Kengne AP, Ashuntantang G. Referral of patients with kidney impairment for specialist care in a developing country of sub-Saharan Africa. Ren Fail. 2009;31(5):341-8.

34. Halle MP, Ashuntantang G, Kaze FF, Takongue C, Kengne A-P. Fatal outcomes among patients on maintenance haemodialysis in sub-Saharan Africa: a 10-year audit from the Douala General Hospital in Cameroon. BMC Nephrol. 2016;17(1):165.

35. Levey AS, Stevens LA, Schmid CH, Zhang YL, Castro AF, Feldman HI, et al. A new equation to estimate glomerular filtration rate. Ann Intern Med. 2009; 150(9):604-12.

36. Kidney Disease: Improving Global Outcomes (KDIGO) CKD Work Group. KDIGO 2012 clinical practice guideline for the evaluation and management of chronic kidney disease. Kidney Int. 2013:3:1-150.

37. Kaze FF, Kengne AP, Pefura Yone EW, NdamFemben NS, Ashuntantang G. Renal function, urinalysis abnormalities and correlates among HIV-infected Cameroonians naive to antiretroviral therapy. Saudi J Kidney Dis Transplant Off Publ Saudi Cent Organ Transplant Saudi Arab. 2013;24(6):1291-7.

38. Naicker S. Burden of end-stage renal disease in sub-Saharan Africa. Clin Nephrol. 2010;74(Suppl 1):S13-6.

39. Assaram S, Magula NP, Mewa Kinoo S, Mashamba-Thompson TP. Renal manifestations of HIV during the antiretroviral era in South Africa: a systematic scoping review. Syst Rev. 2017;13:6.

40. Mbuagbaw J, Jingi AM, Noubiap JJN, Kaze AD, Nansseu JRN, Bigna JJR, et al. Patterns and trends in mortality among HIV-infected and HIV-uninfected patients in a major internal medicine unit in Yaoundé, Cameroon: a retrospective cohort study. JRSM Open. 2016;7(9):2054.

41. Ando M, Yanagisawa N. Epidemiology, clinical characteristics, and management of chronic kidney disease in human immunodeficiency virusinfected patients. World J Nephrol. 2015;4(3):388-95.

42. Flandre $P$, Pugliese $P$, Cuzin L, Bagnis $C$, Tack I, Cabié A, et al. Risk factors of Chronic Kidney Disease in HIV-infected patients. Clin J Am Soc Nephrol. 2011;6(7):1700-7.

43. Bigé N, Lanternier F, Viard J-P, Kamgang P, Daugas E, Elie C, et al. Presentation of HIV-associated nephropathy and outcome in HAART-treated patients. Nephrol Dial Transplant. 2012;27(3):1114-21.

44. Husain NE, Ahmed MH, Almobarak AO, Noor SK, Elmadhoun WM, Awadalla $\mathrm{H}$, et al. HIV-associated nephropathy in Africa: pathology, clinical presentation and strategy for prevention. J Clin Med Res. 2018;10(1):1-8.

45. Okpechi I, Swanepoel C, Duffield M, Mahala B, Wearne N, Alagbe S, et al. Patterns of renal disease in Cape Town South Africa: a 10-year review of a single-centre renal biopsy database. Nephrol Dial Transplant. 2011;26(6):1853-61.

46. da Silva DR, Gluz IC, Kurz J, Thomé GG, Zancan R, Bringhenti RN, et al. Multiple facets of HIV-associated renal disease. Braz J Med Biol Res. 2016; 49(4):e5176

47. Booth JW, Hamzah L, Jose S, Horsfield C, O'Donnell P, McAdoo S, et al. Clinical characteristics and outcomes of HIV-associated immune complex kidney disease. Nephrol Dial Transplant. 2016;31(12):2099-107. 
48. Zemraoui N, Maoujoud O, Belarbi M, Alaaayoud A, Aseraji M, Bahadi A. La référence tardive au néphrologue des patients en insuffisance rénale chronique: fréquence et conséquences. Research. 2014;1:1255.

49. Halle MP, Jimkap NN, Kaze FF, Fouda H, Belley EP, Ashuntantang G. Cost of care for patients on maintenance haemodialysis in public facilities in Cameroon. Afr J Nephrol. 2017;20(1):230-7.

50. Ahuja TS, Grady J, Khan S. Changing trends in the survival of dialysis patients with human immunodeficiency virus in the United States. J Am Soc Nephrol. 2002;13(7):1889-93.

51. Sarfo FS, Keegan R, Appiah L, Shakoor S, Phillips R, Norman B, et al. High prevalence of renal dysfunction and association with risk of death amongst HIV-infected Ghanaians. J Inf Secur. 2013;67(1):43-50.

52. Ibrahim F, Hamzah L, Jones R, Nitsch D, Sabin C, Post FA, et al. Baseline kidney function as predictor of mortality and kidney disease progression in HIV-positive patients. Am J Kidney Dis. 2012;60(4):539-47.

53. Post FA, Campbell $L$, Hamzah L, Collins $L$, Jones R, Siwani R, et al. Predictors of renal outcome in HIV-associated nephropathy. Clin Infect Dis. 2008;46(8):1282-9.

54. Choi Al, Rodriguez RA, Bacchetti P, Volberding PA, Havlir D, Bertenthal D, et al. Low rates of antiretroviral therapy among HIV-infected patients with chronic kidney disease. Clin Infect Dis. 2007;45(12):1633-9.

55. Peters PJ, Moore DM, Mermin J, Brooks JT, Downing R, Were W, et al. Antiretroviral therapy improves renal function among HIV-infected Ugandans. Kidney Int. 2008;74(7):925-9.

56. Perinbasekar S, Brod-Miller C, Pal S, Mattana J. Predictors of survival in HIVinfected patients on hemodialysis. Am J Nephrol. 1996;16(4):280-6.

57. Smart NA, Titus TT. Outcomes of early versus late nephrology referral in chronic kidney disease: a systematic review. Am J Med. 2011;124(11):1073-80.

58. Avorn J, Bohn RL, Levy E, Levin R, Owen WF, Winkelmayer WC, et al. Nephrologist care and mortality in patients with chronic renal insufficiency. Arch Intern Med. 2002;162(17):2002-6.

\section{Publisher's Note}

Springer Nature remains neutral with regard to jurisdictional claims in published maps and institutional affiliations.

Ready to submit your research? Choose BMC and benefit from:

- fast, convenient online submission

- thorough peer review by experienced researchers in your field

- rapid publication on acceptance

- support for research data, including large and complex data types

- gold Open Access which fosters wider collaboration and increased citations

- maximum visibility for your research: over $100 \mathrm{M}$ website views per year

At $\mathrm{BMC}$, research is always in progress.

Learn more biomedcentral.com/submissions 